\title{
Psychophysics of taste lateralization on anterior tongue
}

\author{
HIROKI SHIKATA \\ Monell Chemical Senses Center, Philadelphia, Pennsylvania and \\ Tobacco Science Research Laboratory, Japan Tobacco Inc., Yokohama, Japan \\ DAVID B. T. MCMAHON \\ Monell Chemical Senses Center, Philadelphia, Pennsylvania \\ and University of Pittsburgh, Pittsburgh, Pennsylvania \\ and \\ PAUL A. S. BRESLIN \\ Monell Chemical Senses Center, Philadelphia, Pennsylvania
}

\begin{abstract}
There have been very few investigations of the spatial properties of taste stimuli localized to specific areas of the oral cavity. This is surprising, since the spatial localization of taste sensations may contribute to the overall taste percept, much as do quality, intensity, and the temporal characteristics of tastes. The difficulty in eliminating the confounding factor of a tactile sensation may partially account for the paucity of such studies, since a gustatory stimulus cannot be presented as a liquid without a tactile component. As a step toward understanding the localizability of gustatory sensations, we designed a yoked stimulator and an experimental procedure to control for tactile cues. Lateral discrimination was evaluated at the tip of the tongue with four taste stimuli (sodium saccharin, sodium chloride, citric acid, and quinine hydrochloride) by presenting a taste and a blank solution simultaneously at two locations on the tongue. We found that subjects could lateralize all four taste stimuli in the absence of any discriminative tactile cues. Subjects' ability to lateralize varied as a psychometric function of the stimulus concentration. Detection thresholds, measured in a forced-choice two-interval staircase procedure with the same yoked stimulator that was used in the lateralization task, were always lower than lateralization thresholds, and both lateralization and detection thresholds were correlated within subjects. Subjects were unable to lateralize taste cues on a nongustatory surface under the upper lip at the highest tested concentrations, at which performance was $100 \%$ on a gustatory surface (dorsal anterior tongue). These results show that (1) taste compounds can be lateralized in the absence of any discriminative mechanical cue (but only on the gustatory epithelium) and (2) although the localization of a compound does not logically require conscious detection of the taste (cf. blind sight), subjects always detected a taste when they were able to lateralize.
\end{abstract}

We often experience localized taste stimulation when we eat candy or chew gum and take for granted that we can point out where we feel a taste in the oral cavity. However, such localizations may be predominantly guided by the tactile cue that co-occurs with the taste cue. The localizability of taste cues could be a factor that is as important as quality, intensity, and temporal dynamics to the overall impact of taste perception on behavior. All of these attributes could contribute to the identification and oral manipulation of the sapid bolus and could influence the decision to swallow. Nevertheless, there have been very few studies of the psychophysics of taste localization. Is

The authors thank Caitlin Aptowicz for her excellent technical assistance. This study was supported by Japan Tobacco Inc. and NIH Grant R29 DC02995 (PASB). Correspondence concerning this article should be addressed to P. A. S. Breslin, Monell Chemical Senses Center, 3500 Market St., Philadelphia, PA 19104 (e-mail: breslin@monell.org). there a basis for the spatial perception of taste? One possibility is that gustatory sensations carry spatial information directly; that is, the taste system has its own Ortsinn, in Weber's terminology. An alternative hypothesis is that gustatory percepts are not directly encoded spatially but are interpreted by the brain as originating from regions in the mouth that receive concurrent, discriminative tactile stimulation.

We define the word localization as the ability to obtain reliable spatial information from a sensory signal. The sense of vision clearly enables us to localize a light stimulus, with particularly fine resolution near the fovea (Barlow \& Mollon, 1982). Auditory stimuli are also localizable, since we have a good sense of the lateral direction of sound, which is obtained by the time difference and intensity difference between both ears (Mills, 1958, 1960; Rayleigh, 1909). Localization of front versus back auditory signals is more difficult. The skin senses have excellent localizability for touch (Weinstein, 1968), pain, or, to 
a much lesser degree, thermal sensations (Hensel, 1982; Stevens, 1991). Touch sensations yield the highest spatial resolution of all the somatosensory modalities. In contrast, the olfactory sense in humans has no spatial dimension and does not even enable lateralization of a pure olfactory stimulus (such as vanillin or phenyl ethyl alcohol) to a single nostril, although lateralization of a trigeminal irritant (such as menthol or carbon dioxide) is possible (Kobal, Van Toller, \& Hummel, 1989).

There have been few studies of the localization ability of the taste system, perhaps because of the difficulty in eliminating the confounding factor of touch, since a gustatory stimulus cannot be easily presented as a liquid without a tactile sensation. In the earliest study of taste localization, Skramlik (1924) stimulated several tongue regions simultaneously with various taste solutions, using two or more fine brushes, and found that subjects had the ability to localize taste sensation with good spatial resolution. However, his methods were not sufficiently quantitative for modern psychophysical standards. Moreover, the possibility exists that the two brushes may not have touched the tongue at precisely the same time, which would introduce a confounding tactile difference between the two stimuli that the subjects may have been able to perceive. Bekesy (1964) conducted a detailed study of taste lateralization and tried to understand the phenomenon in a context similar to that for his work on localization of hearing and touch (Bekesy, 1959). He used a lingual stimulator controlled by a mechanical valve that delivered the taste stimulus on the left and right sides of the tongue. He showed lateralization of taste sensations as a function of differences in onset time and intensity between two stimuli. However, his data were based on the introspection of experts, who were thought to be able to separate the confounding tactile sensation, caused by fluctuations in the fluid pressure in the delivery tubes, from the taste sensation. He believed that the pressure sensation of stimulus onset (created by valve switching) was so different from the taste sensation that observers could easily distinguish between them. Thus, his elegant work remains inconclusive, since it is not clear that subjects can successfully disregard the tactile component of a sensory stimulus with both gustatory and mechanical elements.

In contrast, Bartoshuk has proposed that gustatory sensations are not inherently localizable but, rather, that their localization depends on accompanying somatosensory cues (Miller \& Bartoshuk, 1991; Todrank \& Bartoshuk, 1991). The clinical observation that patients with partial ageusia are frequently unaware of the loss of taste sensitivity on one side of the tongue suggests that the tactile components of a food bolus can mediate spatial taste perception via a mechanism of tactile capture (Miller \& Bartoshuk, 1991). A similar taste illusion has been elicited in healthy subjects by dragging a cotton swab (soaked in a taste stimulus) from a fungiform papilla-dense area of the tongue (tip) to an area with few or no taste receptors:
Under these conditions, the subjects frequently reported that the taste sensations followed the swab (Todrank \& Bartoshuk, 1991). These observations suggest that, in cases of conflicting gustatory and tactile cues, the brain biases spatial perception in favor of the tactile input. However, it does not necessarily follow that gustatory stimuli cannot be localized in the absence of discriminatory tactile cues.

It is not generally possible to deliver a taste stimulus without an accompanying tactile cue, because bringing a taste stimulus into contact with the tongue also stimulates mechanoreceptors. We addressed this problem in our experiments by delivering a specific taste stimulus (solution) at a precise region in the oral cavity while giving an identical tactile sensation with pure water at a different region. With this method, we can stimulate two loci with any two stimuli simultaneously, such as a salt solution on the left side of the tongue and water on the right. To implement this procedure, we designed a stimulator that could deliver stimuli to two specific regions of the tongue at the same time with a relatively weak tactile sensation and could control the stimulated area and stimulus volume with good reproducibility. The questions of interest were the following. (1) Can subjects localize (lateralize) taste sensations on the anterior tongue in the absence of discriminatory tactile cues? (2) Does taste localization depend on taste detection? (3) Can localization of gustatory stimuli be dissociated from chemesthetic cues?

\section{GENERAL METHOD}

\section{Subjects}

Ten subjects ( 4 females and 6 males), between the ages of 20 and 42 , were recruited from the Monell staff and were paid to participate in the three experiments. Two were smokers. The subjects were asked to refrain from eating, smoking, or drinking at least $1 \mathrm{~h}$ prior to testing. Before the enrollment in any experiments, the protocol was explained to prospective subjects, who were screened with 10 trials, in which they were required to lateralize $500 \mathrm{mM}$ sodium chloride $(\mathrm{NaCl})$ versus water. The cutoff criterion for accepting subjects was to lateralize $500 \mathrm{mM} \mathrm{NaCl}$ versus water perfectly within 10 trials $(10 / 10)$. One subject who did not discriminate perfectly $(9 / 10$ correct) was excluded, on the assumption that he did not have normal taste sensitivity or was unable to follow instructions. All 10 subjects participated in Experiments 1, 2, and 3.

\section{Apparatus}

We designed the cotton-swab stimulator shown in Figure 1 to deliver the taste stimulus and the blank stimulus simultaneously. This device has two sections, the swab clamp and the stimulus delivery syringes, connected by polyethylene tubing for stimulus delivery. The swab clamp consisted of two cotton swabs held parallel by a plastic holder. The holder was designed to hold swabs with fixed distance and angle (parallel) and to enable the experimenter to detach the swabs easily, so new swabs could be used on each trial. The distance between the swabs was set to $3 \mathrm{~cm}$, in order to have a relatively large separation between stimuli across the midline (Tomita, Ikeda, \& Okuda, 1986) and yet still stimulate high densities of fungiform papillae near the tip of the tongue. This approach was adapted in order to maximize the a priori chances of successfully 

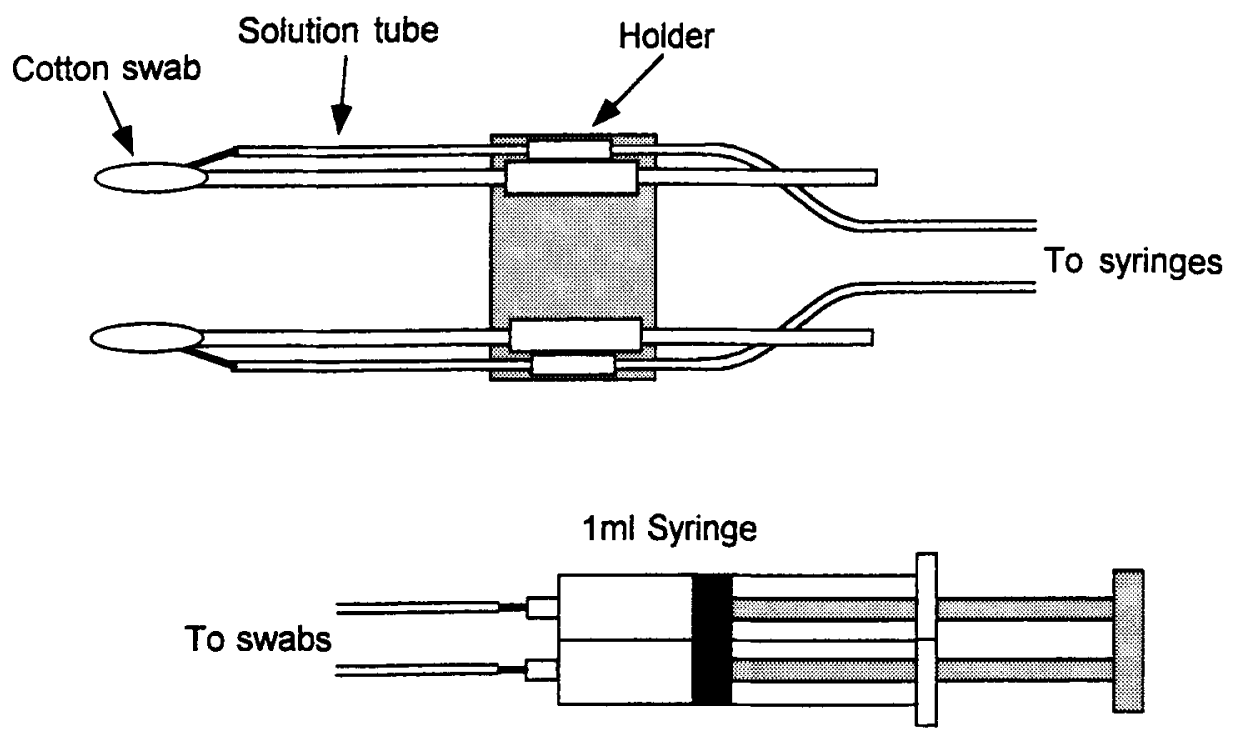

Figure 1. Design of the cotton swab stimulator.

demonstrating lateralization. Solution tubes were inserted into the swabs with $18-\mathrm{g}$ stainless needles to anchor the tubes in the swabs and to guarantee moistening the swabs. The solution delivery section of the device consisted of two 1-ml syringes that were glued together. The plungers of the syringes were connected by a plastic plate so that the same volume of stimulus would be infused at precisely the same time.

The stimulation procedure was as follows: (1) Fill the syringe and the attached solution tube with the stimulus solution on one side and with water on the other side; (2) attach two new cotton swabs to the holder and insert the needles into the swabs; (3) gently apply the dry swabs to the tip of the subject's tongue on the dorsal tip, approximately $8 \mathrm{~mm}$ posterior to the leading edge of the tongue (see Figure 2); and (4) infuse approximately $0.1 \mathrm{ml}$ of the two liquids (taste solution and water diluent) so that they moisten the swabs but do not drip. This protocol enabled us to stimulate both sides of the tongue simultaneously, with no discriminative tactile cues from the stimulus delivery.

The area stimulated by the cotton swabs when infused with $0.1 \mathrm{ml}$ of solution was determined by applying the cotton swabs to the tongue and infusing them with $0.1 \mathrm{ml}$ of blue food coloring in 2 subjects. The resulting stained areas were photographed, and the areas were calculated to be $40^{2} \mathrm{~mm}$, on average (see Figure 2).

\section{Stimuli}

Solutions of sodium saccharin (Na-saccharin), $\mathrm{NaCl}$, citric acid, and quinine hydrochloride (quinine- $\mathrm{HCl}$ ) were used to investigate a broad range of taste qualities. All the compounds were reagent grade and purchased from Sigma $\mathrm{Co}$. The compounds were dissolved in deionized water and maintained at room temperature. The concentrations of the stimuli were defined as the 0.3 or 0.5 log step series, in which the lowest concentration was chosen so that it would be roughly one order of magnitude below the reported whole-mouth detection threshold (Pfaffmann, Bartoshuk, \& McBurney, 1971; see Table 1 for concentration series). The stimuli were selected to mimimize nongustatory cues and to stimulate taste at very low concentrations, to avoid osmotic cues. For example, saccharin was chosen instead of sucrose in order to avoid high concentrations of the sweet stimulus (e.g., $500 \mathrm{mM}$ ) and the consequent osmotic cues, as well as to avoid other physical differences between the stimulus and water, such as viscosity and solution density, that would accompany strong sucrose solutions.

Deionized water was used for the blank stimulus and the rinsing agent in all the experiments. In each trial, several yoked stimulators were employed.

\section{EXPERIMENT 1}

In Experiment 1, we investigated whether subjects could lateralize the taste stimuli in the absence of a discriminative tactile cue.

\section{Method}

Procedure. Before the experimental session, each subject was asked to rinse and expectorate with deionized water three times. For each trial, he or she was requested to stick out his or her tongue and to seal his or her lips around the tongue. A cotton swab stimulator was presented, and the subject was required to indicate on which side he or she perceived a stimulus by raising his or her right or left hand. The stimulus was presented for $5 \mathrm{sec}$, and the subject was not allowed to withdraw the tongue until he or she responded. The subject was given feedback on each trial regarding whether his or her response was correct or incorrect. The subject rinsed his or her mouth at least twice in the 1-min interval between trials. A session consisted of 12 trials, including 2 blank trials with water presented to both sides. Blank trials were employed to test for biases and side preferences in the subjects. The concentration of the stimulus was constant within a test session, and the order of stimulus side was chosen randomly and counterbalanced. The order of chemical compounds tested was also randomized, but the order of concentrations for each compound was descending so that the subject could initially identify the quality of the localized taste. Each subject participated in one session per day and from four to five sessions per week, until he or she had at least 20 trials for each concentration for each compound. Thus, there was a minimum of 680 trials per subject.

Analysis. Since the procedure had a two-alternative, forcedchoice design and each trial was independent of the preceding trials, the data were treated as Bernoulli trials. Thus, we employed a chi- 


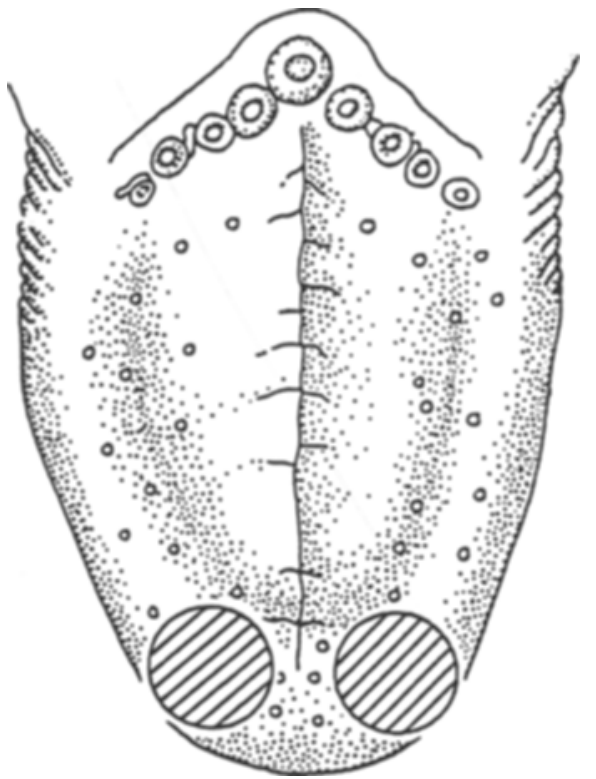

Figure 2. The areas on the dorsal tongue surface stimulated by two solutions presented via the yoked stimulator. The cotton swabs were applied to the region approximately $8 \mathrm{~mm}$ posterior to the leading edge of the tongue, $3 \mathrm{~cm}$ apart from each other. Approximately $0.1 \mathrm{ml}$ of fluid was injected into each swab simultaneously. The stimulated area was approximately $40 \mathrm{~mm}^{2}$ for each swab. From Sensation and Perception (2nd ed., p. 425), by E. B Goldstein, 1984, Belmont, CA: Wadsworth. Copyright 1984 by Wadsworth. Adapted with permission.

square test to the hypothesis that $p$ (discrimination probability) $=.5$ for each data point.

Furthermore, to characterize the whole discrimination functions, the data were fitted with a logistic function described below;

$$
p(K)=.5\left\{1+\frac{1}{1+\exp \left[\frac{-(\log K-\log \mu)}{\log \sigma}\right]}\right\} \text {. }
$$

In Equation 1, $K$ is the stimulus concentration, $\mu$ is the inflection point of the curve, $\sigma$ represents the steepness of the curve, and $p$ is the probability of answering correctly. The parameter $\mu$ also provides the concentration where $p(K)=.75$, which we defined as the threshold for lateralization of the taste. The parameter $\sigma$ can be used to obtain a Weber fraction for lateralization from the discrimination profiles, as will be described below. Curve fitting was performed by nonlinear least squares regression.

These analyses were performed on the arithmetic mean data from all the subjects, as well as on the data from each individual.

\section{Results}

Figures 3 and 4 show the mean and the individual data, respectively, for each compound. In each case, the subjects could lateralize the stimulus as a psychometric function of stimulus concentration. The subjects' lateralization performance declined as the stimulus concentration decreased. The functions of both the group and the individual curves were closely fit by the logistic function. The parameter $\mu$, which represents the threshold of lateralization for each compound, is shown in Table 2 and is compared with the detection thresholds by whole mouth (Bartoshuk, Rifkin, Marks, \& Bars, 1986; Pfaffmann et al., 1971; Stevens, 1995). Although detection thresholds for small portions of the anterior tongue are available for comparison, the researchers' methods were sufficiently different from the present methods to prevent meaningful comparison (Collings, Lindberg, \& McBurney, 1976; Linschoten \& Kroeze, 1994). Not surprisingly, the lateralization thresholds were slightly higher than the older detection thresholds and considerably higher than the more recent whole-mouth thresholds. However, the relative rankings of sensitivity to the compounds were consistent across studies.

From Equation 1, we can obtain the Weber fraction as $\Delta K / K=2.303\left(\log _{10} \sigma\right)$, where $K=\mu$ and $\Delta K$ is the discrimination threshold, taking the $87.5 \%$ discrimination level as the Weber threshold criterion (derived from Breslin, Beauchamp, \& Pugh, 1996). The Weber fraction for each compound is also shown in Table 2 and is compared with previously reported values (Pfaffmann et al., 1971). The Weber fractions for lateralization were from three to five fold larger than those based on whole-mouth stimulation, which suggests that the punctate area lateralization task is more difficult than a whole-mouth detection task.

\section{EXPERIMENT 2}

In Experiment 2, we measured the subjects' absolute detection thresholds for each compound, using the same yoked apparatus and procedure as those in Experiment 1.

\section{Method}

Procedure. The same cotton swab stimulator was used for presenting stimuli as that in Experiment 1 . In this experiment, a trial consisted of two stimulator presentations, a target and a blank. In the target presentation, the subject was presented with a stimulus on the left or right side of the tongue while water was simultaneously presented on the other side. In the blank presentation, the subject received water on both sides. The subject was required to respond simply by identifying in which presentation interval the target was

Table 1

Concentration Series for the Stimuli in Experiment 1

\begin{tabular}{|c|c|c|c|c|c|c|c|c|}
\hline Stimulus & & & Con & ntration & $(\mathrm{mm}) \mathrm{s}$ & eries & & \\
\hline $\mathrm{Na}-$ Saccharin & 0.00 & 0.01 & 0.025 & 0.1 & 0.25 & 0.5 & 1.0 & 5.0 \\
\hline $\mathrm{NaCl}$ & 2.5 & 5.0 & 10.0 & 25.0 & 50.0 & 100.0 & 250 & 500 \\
\hline Citric & 0.01 & 0.032 & 0.1 & 0.32 & 1.0 & 3.2 & 10.0 & 32.0 \\
\hline Quinine- $\mathrm{HCl}$ & 0.001 & 0.0032 & 0.01 & 0.032 & 0.1 & 0.32 & 1.0 & 3.2 \\
\hline
\end{tabular}




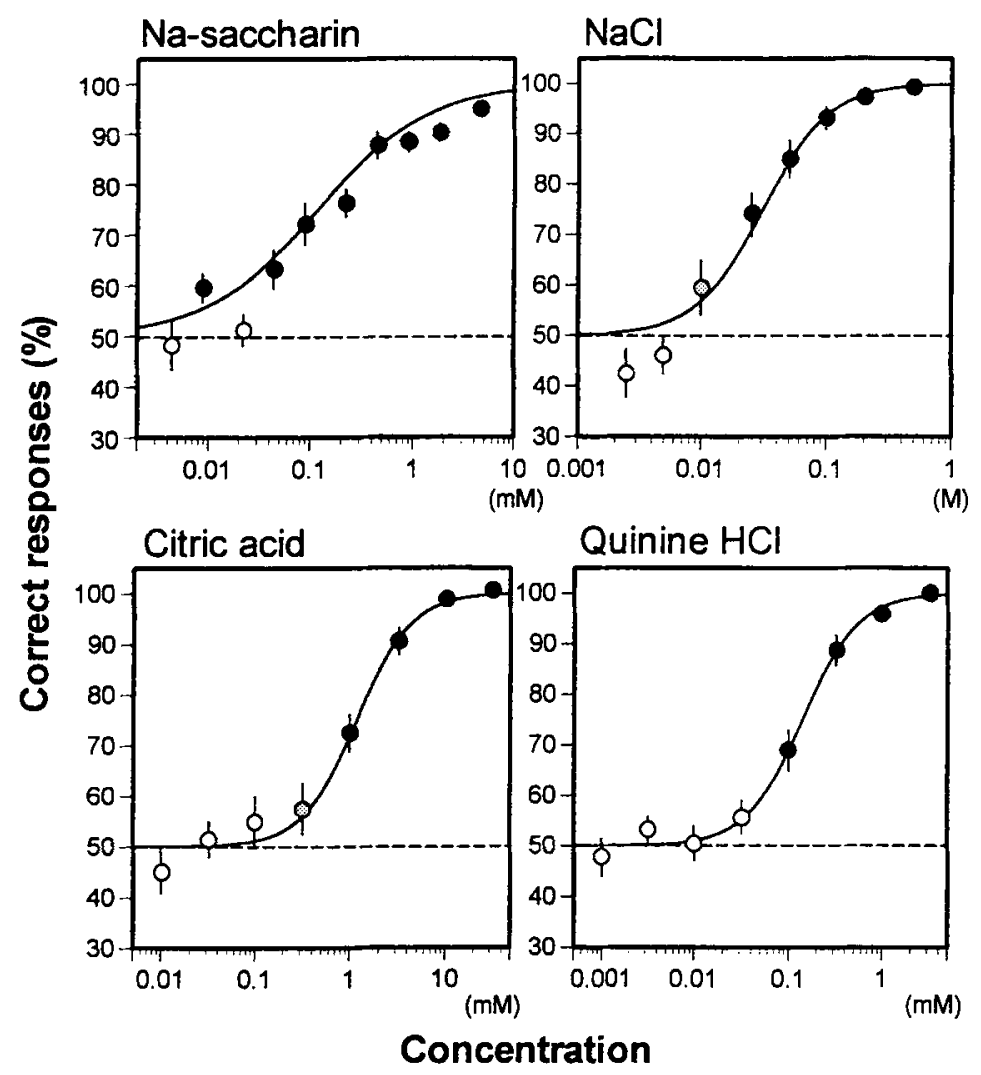

Figure 3. Response curves of lateralization for each compound. Each plot represents mean $\pm S E M$ value across $>\mathbf{2 0 0}$ trials. Filled circles identify the data points significantly different from chance $(p<.01)$; gray circles identify the points weakly different from chance $(.01<p<.05)$; open circles identify the points not significantly different from chance $(p<.05)$. Curve fittings were made by logistic functions (Equation 1).

presented; the lateral side of the target was irrelevant. The order of target presentation and stimulus side was randomized. There was a 10 -sec pause between the two presentations, during which the subject's tongue was rinsed by the investigator with deionized water from a squeeze bottle. The subject was not permitted to withdraw the tongue into the mouth until after the second interval, whereupon he or she indicated in which interval the stimulus was presented. After responding, the subject was required to rinse the whole mouth twice with deionized water prior to the next trial. The threshold was obtained by an up-down staircase method (Cornsweet, 1962) according to the two-out-of-three rule, as will be described below. The detection threshold was calculated from the geometric mean of last six consecutive reversals. One detection threshold was measured for each subject and for each compound.

Two-out-of-three rule. Since there might be a slight difference of sensitivity between the two sides of the tongue, we had to be careful when measuring the absolute detection threshold of a lateralized taste. If a stimulus was presented on a less sensitive side, a higher detection threshold value would be obtained than from the other side. The two-out-of-three rule is a modified up-down staircase procedure that enabled us to test the potentially more sensitive side at each concentration step. The rule has three principles: (1) The stimulus concentration is lowered after two out of three correct responses $(++,-++,+-+)$; (2) when a subject responds incorrectly $(-)$, the stimulus target is presented on the other side of the tongue in the next trial at the same concentration; and (3) stimulus concentration is raised after two incorrect responses $(--$, $+--,-+-)$. The concentration range within which all reversals were accepted was clamped at 1.25 orders of magnitude. Therefore, if a subject deviated from this range more than two concentration series steps from their first reversal, in either increasing or decreasing concentration, the counting of reversals would begin again. These rules collectively enable the detection threshold to be determined for the more sensitive side of the tongue, if there is a more sensitive side. And the threshold would be determined as a simple two-downone-up rule from the perspective of the sensitive side. The convergence point for this rule with two-alternative forced-choice procedure is 0.75 , which means it is possible to compare the detection thresholds with lateralization thresholds for which the convergence point is also 0.75 by its definition. The subjects were not aware of the decision rules that were followed for measurement of this detection threshold.

\section{Results}

Table $3 \mathrm{~A}$ shows the average detection threshold for each compound, as compared with the lateralization threshold. The detection thresholds were about an order of magnitude lower than the lateralization thresholds, and their relative ranking was consistent, with the excep- 

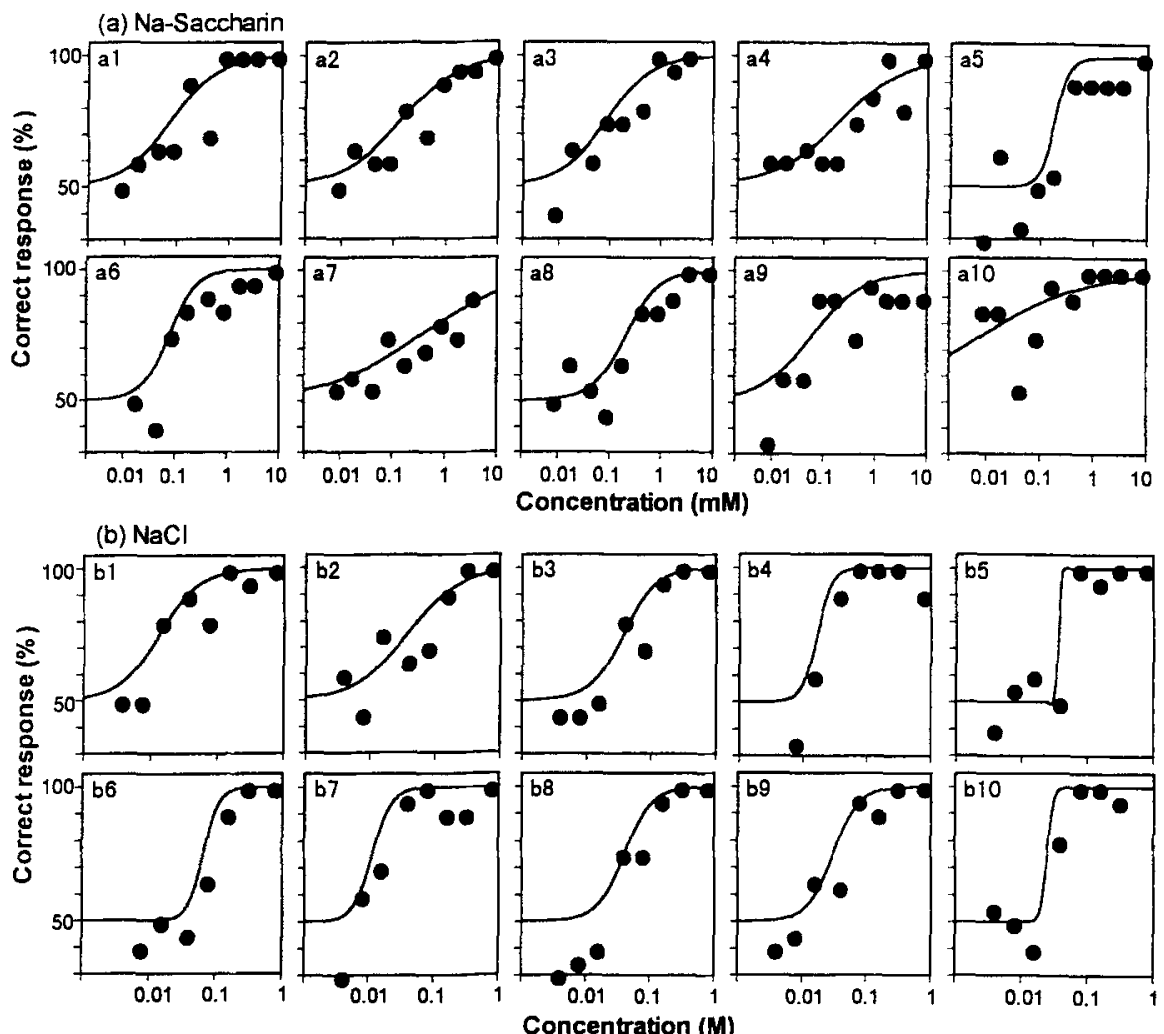

(c) Citric acid
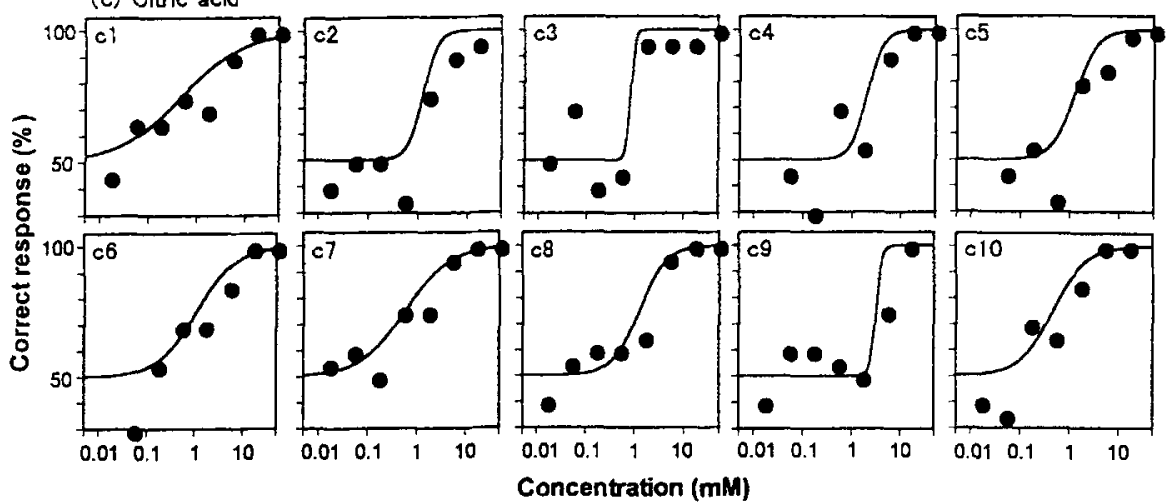

(d) Quinine $\mathrm{HiCl}$
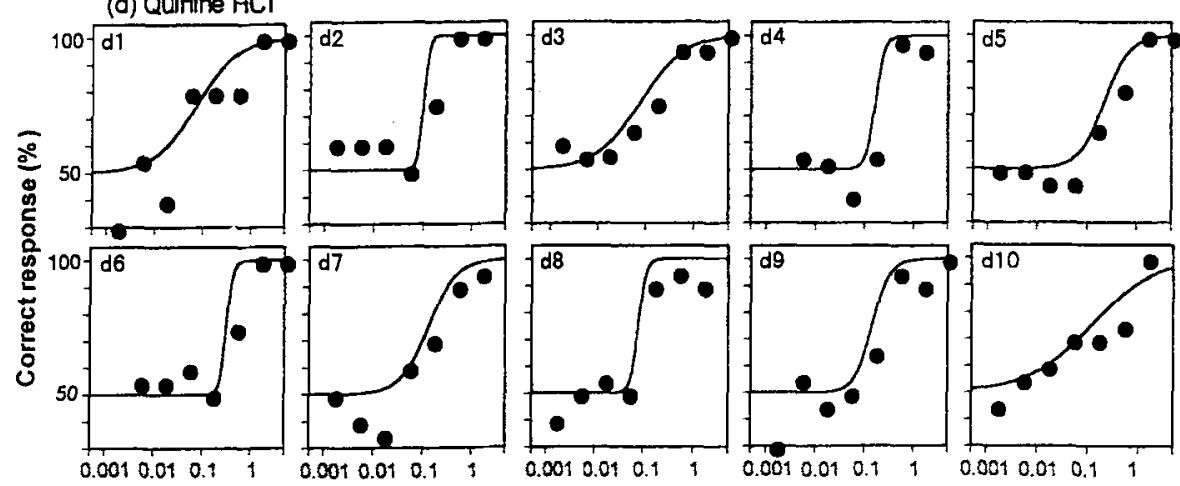

Concentration (mM)

Figure 4. Individual plots of correct responses for taste lateralization against stimulus concentration. The title of each plot $(\mathbf{a} 1, \mathrm{a} 2, \ldots \mathrm{d} 10)$ corresponds to Table 1. 
Table 2

Lateralization Thresholds $(\mu)$ and Weber Fractions $(\Delta K / K)$ for Each Subject and Each Compound

\begin{tabular}{|c|c|c|c|c|c|c|c|c|}
\hline Subject & Figure & $\mu(\mathrm{mM})$ & $\sigma$ & $\Delta K / K$ & Figure & $\mu(\mathrm{mM})$ & $\sigma$ & $\Delta K / K$ \\
\hline \multicolumn{5}{|c|}{ Na-Saccharin } & \multicolumn{4}{|c|}{$\mathrm{NaCl}$} \\
\hline 1 & al & 0.07 & 2.97 & 1.09 & bl & 13 & 2.02 & 0.70 \\
\hline 2 & a2 & 0.13 & 3.60 & 1.28 & b2 & 37 & 2.55 & 0.94 \\
\hline 3 & a3 & 0.08 & 2.80 & 1.03 & b3 & 37 & 1.83 & 0.60 \\
\hline 4 & a4 & 0.20 & 4.35 & 1.47 & b4 & 16 & 1.32 & 0.28 \\
\hline 5 & a5 & 0.18 & 1.38 & 0.32 & b5 & 35 & 1.05 & 0.04 \\
\hline 6 & a6 & 0.08 & 1.83 & 0.61 & b6 & 66 & 1.31 & 0.27 \\
\hline 7 & a7 & 0.34 & 8.40 & 2.13 & b7 & 11 & 1.42 & 0.35 \\
\hline 8 & a8 & 0.21 & 2.10 & 0.74 & b8 & 40 & 1.64 & 0.50 \\
\hline 9 & a9 & 0.06 & 3.20 & 1.16 & b9 & 30 & 1.60 & 0.47 \\
\hline 10 & al0 & 0.01 & 10.23 & 2.33 & b10 & 24 & 1.12 & 0.11 \\
\hline & & $0.10^{*}$ & & $1.22^{\dagger}$ & & $27^{*}$ & & $0.43^{+}$ \\
\hline Pfaffmann & & 0.02 & & 0.27 & & 10 & & 0.15 \\
\hline Bartoshuk & & & & & & 0.90 & & \\
\hline \multicolumn{2}{|l|}{ Stevens } & & & & & 0.89 & & \\
\hline \multicolumn{5}{|c|}{ Citric acid } & \multicolumn{4}{|c|}{ Quinine- $\mathrm{HCl}$} \\
\hline 1 & $\mathrm{cl}$ & 0.48 & 4.66 & 1.54 & dl & 0.07 & 2.75 & 1.01 \\
\hline 2 & $\mathrm{c} 2$ & 1.22 & 1.42 & 0.35 & $\mathrm{~d} 2$ & 0.10 & 1.15 & 0.14 \\
\hline 3 & c3 & 0.79 & 1.12 & 0.11 & $\mathrm{~d} 3$ & 0.07 & 2.87 & 1.05 \\
\hline 4 & c4 & 1.95 & 1.47 & 0.38 & $\mathrm{~d} 4$ & 0.16 & 1.24 & 0.21 \\
\hline 5 & c5 & 1.23 & 1.71 & 0.54 & d5 & 0.22 & 1.78 & 0.58 \\
\hline 6 & c6 & 1.08 & 2.57 & 0.94 & d6 & 0.32 & 1.17 & 0.16 \\
\hline 7 & c7 & 0.57 & 2.87 & 1.05 & d7 & 0.13 & 1.91 & 0.65 \\
\hline 8 & c8 & 1.26 & 1.89 & 0.64 & d8 & 0.08 & 1.19 & 0.18 \\
\hline 9 & c9 & 3.20 & 1.15 & 0.14 & d9 & 0.14 & 1.51 & 0.41 \\
\hline 10 & $\mathrm{cl} 10$ & 0.47 & 1.27 & 0.78 & d10 & 0.12 & 4.26 & 1.45 \\
\hline$M$ & & $1.03^{*}$ & & $0.65^{\dagger}$ & & $0.13^{*}$ & & $0.58^{\dagger}$ \\
\hline Pfaffmann & & 0.73 & & 0.21 & & 0.03 & & 0.21 \\
\hline Bartoshuk & & 0.020 & & & & 0.0010 & & \\
\hline Stevens & & 0.032 & & & & 0.0016 & & \\
\hline
\end{tabular}

Note $-\mu$ and $\sigma$ are the least squares curve fitting parameters of Equation 1, calculated from the individual responses. The last rows represent the whole-mouth detection thresholds and Weber fractions adopted from Pfaffmann, Bartoshuk, and McBurney (1971), Bartoshuk, Rifkin, Marks, and Bars (1986), and Stevens (1995). *Geometric mean. †Arithmetic mean.

tion of a reversal between saccharin and quinine- $\mathrm{HCl}$. Figure 5 shows the correlation between lateralization threshold and detection threshold for each compound. A strong positive correlation was observed for citric acid $(r=.73, p=.02)$ and quinine- $\mathrm{HCl}(r=.93, p=.0001)$, a weak positive correlation for $\mathrm{NaCl}(r=.50, p=.14)$, and no significant correlation was seen for saccharin $(p>.2)$. Note that the threshold measurement was made only once for each subject for each compound, which means the estimation of threshold values was less precise than had we measured thresholds multiple times (Stevens, 1995). Nevertheless, these results suggest that, for at least some taste compounds, there is a close relation between detection and lateralization, with detection showing greater sensitivity.

\section{EXPERIMENT 3}

In Experiment 3, we investigated whether the subjects could lateralize a taste stimulus when it was presented to the nongustatory oral epithelium, in order to control for nongustatory discrimination cues, such as irritation or thermal cues.

\section{Method}

Stimuli. The taste stimuli presented were $5 \mathrm{mM}$ saccharin, $500 \mathrm{mM} \mathrm{NaCl}, 32 \mathrm{mM}$ citric acid, and $3.2 \mathrm{mM}$ quinine- $\mathrm{HCl}$, the highest concentrations employed in Experiment 1 for each compound. At these concentrations, all the subjects could lateralize the stimuli with $100 \%$ accuracy on the tip of the tongue.

Procedure. The procedure was the same as that in Experiment 1, except that the stimulating position was the upper gum under the upper lip. The subjects participated in 20 trials for each compound.

\section{Results}

Table 4 shows the number of correct responses for lateralization on the gums. All the responses were around $50 \%$ and were not different from chance. This failure to lateralize suggests that localization in Experiment 1 was primarily based on the presence of taste cues and was dependent on stimulation of gustatory epithelium.

\section{DISCUSSION}

The purpose of this study was to explore whether taste sensations contained topographical information, in addition to temporal, quality, and intensity information. As a specialized skin sense, one might expect taste sensations 
Table 3A

Lateralization and Detection Thresholds for Each Compound

\begin{tabular}{lcc}
\hline Compound & Lateralization Threshold $(\mathrm{mM})$ & Detection Threshold $(\mathrm{mM})$ \\
\hline Saccharin & $0.097 \pm 0.066$ & $0.015 \pm 0.010$ \\
$\mathrm{NaCl}$ & $27.0 \pm 9.7$ & $3.9 \pm 2.0$ \\
Citric acid & $1.03 \pm 0.40$ & $0.085 \pm 0.083$ \\
Quinine- $\mathrm{HCl}$ & $0.13 \pm 0.04$ & $0.0065 \pm 0.0070$ \\
\hline
\end{tabular}

Note-Lateralization thresholds were calculated from the least squares curve fitting functions of correct responses (see Table 2), and detection thresholds were obtained by an up down staircase method. Each group value represents the geometric mean \pm $S E M$ for 10 subjects.

to be localizable, since all somatosensory modalities are localizable to varying degrees. Touch sensations provide high-resolution spatial information (Weinstein, 1968), and low intensity thermal sensations provide low-resolution spatial information, owing to high spatial summation and integration (Hensel, 1982; Stevens, 1991). The spatial resolution of both of these modalities vary as a function of the body part stimulated. Somatosensation may be contrasted with olfaction, which does not provide any conscious spatial information (Kobal et al., 1989). Subjects can not even identify the nostril into which an odorant has been introduced. Therefore, the question was posed whether taste would be like olfaction, another chemical sense that has no spatial component to its sensory signal, or like all the other skin senses, which invariably provide some localizable signal. We found that tastes could be localized to the lateral side of the tongue that was stimulated and, therefore, show more similarity to somatosensory modalities than to olfaction, with regard to spatial information present in the sensory signal. The spatial resolution of the localizable component of taste cues has yet to be determined.

\section{Lateralization and Detection}

The cotton swab stimulator delivered two simultaneous stimuli with identical somatosensory properties but different taste properties to the two different loci on the anterior tongue. Therefore, the lateralization functions observed in Experiment 1 could be assumed to reflect subjects' abilities to identify the location of any of the four taste stimuli in the absence of any discriminative tactile or thermal cues. Furthermore, the fact that lateralization data varied as a smooth logistic function of stimulus concentration indicates that the subjects were discriminating the stimuli only by their tastes, since chemesthetic cues would not vary smoothly over these small concentration levels. Across this range of concentrations, the potential for discriminative chemesthetic (irritation) information would differ for each compound and would be present, even for the more irritating chemicals (e.g., $\mathrm{NaCl}$ and citric acid), only at the highest concentrations employed. Note that lateralization was significant at $0.01 \mathrm{mM} \mathrm{Na}-$ saccharin, $10 \mathrm{mM} \mathrm{NaCl}, 0.3 \mathrm{mM}$ citric acid, and $0.1 \mathrm{mM}$ quinine- $\mathrm{HCl}$ (see Figure 3). The thresholds for lateralization were compared with the previously reported wholemouth detection thresholds (Bartoshuk et al., 1986;
Pfaffmann et al., 1971; Stevens, 1995). Although the lateralization thresholds were higher than whole-mouth thresholds, there was no difference in the relative ranking of humans' sensitivities across compounds.

In Experiment 2, the subjects received a lateralized stimulus and water in the yoked stimulator during the target interval, as had been presented in Experiment 1, and two water stimuli during the blank interval. The subjects were required simply to detect the target interval, but not on which side the target was presented during that interval. The detection thresholds were lower than the lateralization thresholds and were comparable with modern wholemouth detection thresholds (see Tables 1 and 2). These data suggest that, just as it is possible to detect a taste at threshold without awareness of its quality, it is possible to detect a taste without awareness of its location in the oral cavity.

The lateralization thresholds were compared with the absolute detection thresholds, to understand the relationship between taste detection and taste lateralization. In order to make a fair comparison between lateralization thresholds and detection thresholds, we had to use the same yoked-swab apparatus for both, because small punctate stimulation may not be comparable with whole-mouth stimulation (the method used for most previously published detection thresholds). As with whole-mouth detection, we found that the detection thresholds were lower than the lateralization thresholds and that the relative ranking of the thresholds was the same. The correlation between lateralization and detection thresholds revealed that subjects who showed low detection thresholds were identified as the ones who showed low lateralization

Table 3B

Individual Detection Thresholds (mM)

\begin{tabular}{ccccc}
\hline & \multicolumn{4}{c}{ Compound } \\
\cline { 2 - 5 } Subject & Na-Saccharin & $\mathrm{NaCl}$ & Citric Acid & Quinine-HCl \\
\hline 1 & 0.0071 & 5.0 & 0.026 & 0.0038 \\
2 & 0.0035 & 2.6 & 0.56 & 0.0018 \\
3 & 0.0071 & 1.8 & 0.012 & 0.00047 \\
4 & 0.0082 & 1.3 & 0.47 & 0.0032 \\
5 & 0.022 & 2.7 & 0.46 & 0.083 \\
6 & 0.040 & 14 & 0.042 & 0.12 \\
7 & 0.013 & 1.3 & 0.024 & 0.024 \\
8 & 0.11 & 6.5 & 0.038 & 0.0024 \\
9 & 0.045 & 5.0 & 0.56 & 0.0068 \\
10 & 0.0073 & 1.6 & 0.024 & 0.0032 \\
\hline
\end{tabular}




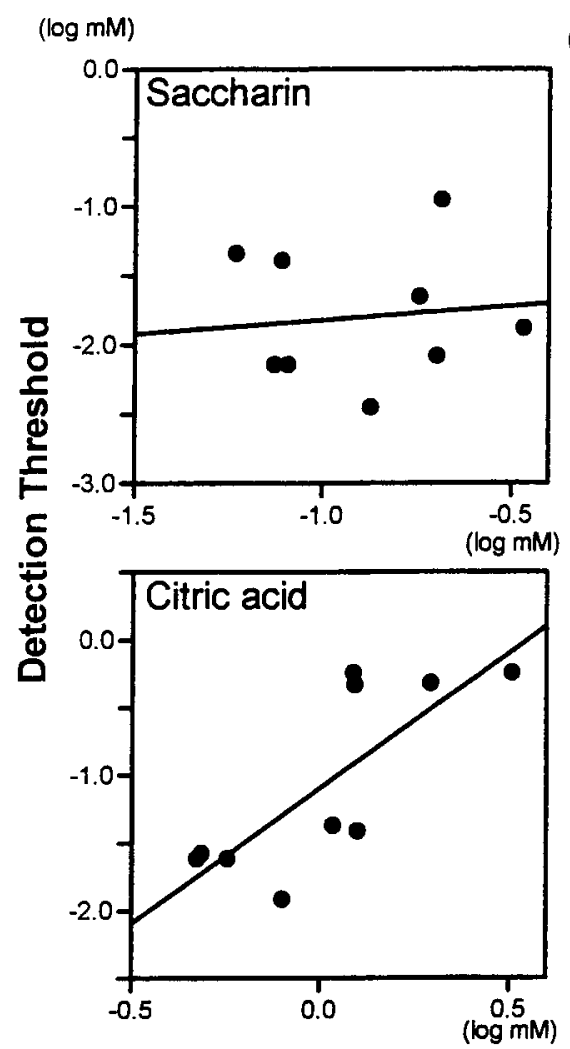

$(\log \mathrm{mM})$
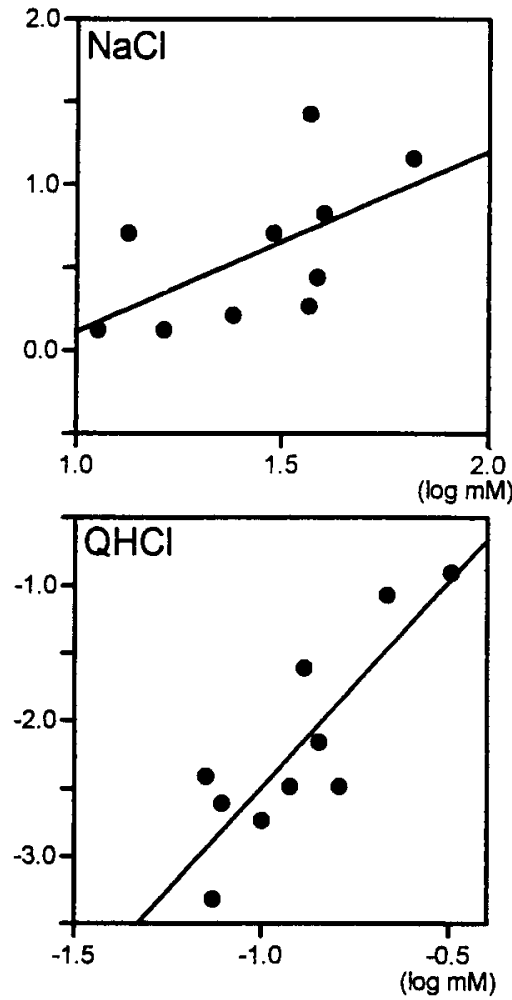

Lateralization threshold

Figure 5. Correlations between lateralization thresholds and detection thresholds. Each plot represents individual threshold values.

thresholds, and vice versa. This suggests that the ability to localize a taste stimulus is associated with the ability to detect the taste. This is further supported by Experiment 3, which clearly showed that stimulus localization was not possible in the absence of taste receptors when tested on the nongustatory epithelium under the upper lip. These results all support the hypothesis that taste lateralization is achieved exclusively via the taste system.

It is important to emphasize this point, because it is not necessary for subjects to detect tastes consciously when they lateralize taste cues, since there are two hypotheses that do not require conscious taste to account for taste localization. First, the lateralization could be indirectly related to taste cues and directly dependent on the stimulus' ability to stimulate irritation signals that we know are localizable. We have ruled out this hypothesis for the reasons given above. Second, it is possible that taste cues could be localizable without conscious awareness that a taste was present. This is analogous to the blind sight phenomenon of geniculostriate-damaged patients who can correctly localize a point light source in front of them when guessing, despite their inability to report seeing any light source at all (Milner, 1998). This hypothesis has also been ruled out in Experiments 2 and 3.

In Experiment 3, the inability of subjects to lateralize the highest concentrations of the stimuli on the upper gum can only be accepted as a demonstration that localization is based on gustatory cues if the tongue tip and the upper gum have comparable chemesthetic sensitivities. This information is not available for the four compounds employed. But there exist data comparing the lip with the tongue tip for sensitivity to capsaicin and piperine. Lawless and Stevens (1988) found that, when stimulating the lip, reports of irritation from capsaicin and piperine were $80 \%$ and $40 \%$, respectively, of the magnitudes at the tongue tip. Therefore, one could conclude that the lip (and possibly the upper gum) is not greatly inferior to the tongue tip for sensing chemical irritation. Thus, the inability of the subjects in the present study to lateralize the four compounds at their highest concentrations on the upper gum supports a gustatory basis for lateralization on

Table 4

Correct Responses for Lateralization on the Upper Gum Under the Upper Lip

\begin{tabular}{lcc}
\hline Compound & Concentration $(\mathrm{mM})$ & Correct Response $(\%)$ \\
\hline Saccharin & 5 & $46.0 \pm 3.4$ \\
$\mathrm{NaCl}$ & 500 & $50.5 \pm 6.7$ \\
Citric acid & 32 & $50.0 \pm 2.9$ \\
Quinine-HCl & 3.2 & $50.0 \pm 4.5$ \\
\hline
\end{tabular}

Note-Each value represents the mean $\pm S E M$ for 10 subjects. None was significantly greater than chance (chi-square analysis). 
the tongue tip. However, these arguments do not entirely eliminate the possibility that chemesthetic cues are playing a role in lateralization on the tongue tip.

In conclusion, (1) taste localization is possible in the absence of discriminatory tactile cues and appears to be dependent on stimulation of the taste reception system. (2) Conscious taste sensations are necessary before the stimulus can be localized. That is, we did not find a phenomenon in our subjects parallel to blind sight, in which subjects are not consciously aware of a light stimulus yet are able to localize the light under forced-choice conditions. And (3) several arguments are made that taste localization is possible in the absence of obvious chemesthetic cues.

\section{Possible Mechanisms of Taste Localization}

From these experimental results, one could make an analogy between gustatory localization and somatosensory localization. In the context of skin senses, the localization of taste resembles the localization of thermal sensations, which requires greater intensity to localize than is required simply to detect thermal changes (Stevens, 1991; Taus, Stevens, \& Marks, 1975), rather than the localization of touch, which seems to be independent of suprathreshold touching pressure (Cholewiak \& Collins, 1991). However, although it is possible to explain the localization mechanisms of the skin senses from their topographical homuncular projections in the cerebral cortex, the mechanisms of taste localization are not known. We have three hypotheses for the mechanisms of taste localization: (1) an oral-lingual gustotopic map in the brain, (2) taste-induced tactile perception, and (3) polymodal taste fibers that are also sensitive to touch and may join into the somatotopic sensory maps. The first hypothesis posits a neural brain map that relates gustatory receptors to their orolingual position, enabling localization of stimulated taste receptors, analogous to the somatotopic or retinotopic neural maps. The second hypothesis posits that when a taste receptor cell is activated by a taste stimulus, it in turn activates adjacent tactile fibers, and the induced secondary tactile signal enables one to localize the stimulated region (Farbman \& Mbiene, 1991; Whitehead \& Kachele, 1994). The third hypothesis recognizes that most taste fibers are also mechanically and thermally sensitive (Hanamori, Hirota, \& Ishiko, 1990; Matsuo et al., 1995; Ogawa, Hayama, \& Yamashita, 1986; Ogawa, Imoto, \& Hayama, 1984; Robinson, 1988; Travers \& Norgren, 1995). Therefore, taste stimulation may project as a somatosensory signal at the same time that it projects to the gustatory cortex via activation of the same primary fibers. There is no neurophysiological evidence to support one hypothesis more than another at present, and each could explain our experimental results. In his electrophysiological explorations of cat nerves, Robinson found that more than $80 \%$ of gustatory chorda tympani nerve fibers were also mechanically sensitive. He proposed that this dual nature of taste fibers might specifically enable the localization of gustatory stimuli. Future studies will focus on distinguishing among these three hypotheses with psychophysical approaches. For example, the use of a lateralization task that employs two qualitatively different but equally intense taste stimuli simultaneously would test the second and third hypotheses.

\section{REFERENCES}

Barlow, H. B., \& Mollon, J. D. (1982). The senses (pp. 133-162). New York: Cambridge University Press.

Bartoshuk, L. M., Rifkin, B., Marks, L. E., \& Bars, P. (1986). Taste and aging. Journal of Gerontology, 41, 51-57.

BEKESY, G. vON (1959). Similarities between hearing and skin sensations. Psychological Review, 66, 1-22.

BEKESY, G. VON (1964). Rhythmical variations accompanying gustatory stimulation observed by means of localization phenomena. Journal of General Physiology, 47, 809-825.

Breslin, P. A. S., Beauchamp, G. K., \& Pugh, E. N., JR. (1996). Monogeusia for fructose, glucose, sucrose, and maltose. Perception \& Psychophysics, 58, 327-341.

Cholewiak, R., \& Collins, A. (1991). Sensory and physiological bases of touch. In M. A. Heller \& W. Schiff (Eds.), The psychology of touch (pp. 23-60). Hillsdale, NJ: Erlbaum.

Collings, V. B., Lindberg, L., \& McBurney, D. H. (1976). Spatial interactions of taste stimuli on the human tongue. Perception \& Psychophysics, 19, 69-71.

CORnSwEET, T. N. (1962). The staircase method in psychophysics. American Journal of Psychology, 75, 485-491.

Farbman, A. I., \& Mbiene, J. P. (1991). Early development and innervation of taste bud-bearing papillae on the rat tongue. Journal of Comparative Neurology, 304, 172-186.

Goldstein, E. B. (1984). Sensation and perception. Belmont, CA: Wadsworth.

HANAMORI, T., Hirota, K., \& Ishiko, N. (1990). Receptive fields and gustatory responsiveness of frog glossopharyngeal nerve: A single fiber analysis. Journal of General Physiology, 95, 1159-1182.

HENSEL, H. (1982). Thermal sensation and thermoreceptors in man. Springfield, Il: Thomas.

Kobal, G., Van Toller, S., \& Hummel, T. (1989). Is there directional smelling? Experimentia, 45, 130-131.

LAWLESS, H. T., \& STEVENS, D. A. (1988). Responses by humans to oral chemical irritants as a function of locus of stimulation. Perception $\&$ Psychophysics, 43, 72-78.

Linschoten, M. R. I., \& Kroeze, J. H. A. (1994). Ipsi- and bilateral interactions in taste. Perception \& Psychophysics, 55, 387-393.

Matsuo, R., Inoue, T., Masuda, Y., Nakamura, O., Yamauchi, Y., \& MORIMOTO, T. (1995). Neural activity of chorda tympani mechanosensitive fibers during licking behavior in rats. Brain Research, 689, 289-298.

Miller, I. J., \& Bartoshuk, L. M. (1991). Taste perception, taste bud distribution, and spatial relationships. In T. V. Getchell, R. L. Doty, L. M. Bartoshuk, \& J. B. Snow, Jr. (Eds.), Smell and taste in health and disease (pp. 205-233). New York: Raven.

MiLls, A. W. (1958). On the minimum audible angle. Journal of the Accoustical Society of America, 30, 237-246.

MilLs, A. W. (1960). Lateralization of high-frequency tones. Journal of the Accoustical Society of America, 32, 132-134.

MiLNER, A. D. (1998). Streams and consciousness: Visual awareness and the brain. Trends in Cognitive Sciences, 2, 25-30.

Ogawa, H., Hayama, T., \& Yamashita, Y. (1986). Thermal sensitivity of taste neurons in solitary tract nucleus of rats. Journal of the Physiological Society of Japan, 48, 309.

Ogawa, H., Imoto, T., \& Hayama, T. (1984). Responsiveness of solitarioparabrachial relay neurons to taste and mechanical stimulation applied to the oral cavity in rats. Experimental Brain Research, 54, 349 358

Pfaffmann, C., Bartoshuk, L. M., \& McBurney, D. H. (1971). Taste psychophysics. In L. M. Beidler (Ed.), Handbook of sensory physiology (Vol. 4, pp. 76-98). New York: Springer-Verlag.

RAYLEIGH, O. M. (1909). On the perception of the direction of sound. Proceedings of the Royal Society of London: Series A, 83, 61-64. 
RoBINSON, P. P. (1988). The characteristics and regional distribution of afferent fibres in the chorda tympani of the cat. Journal of Physiology, 406, 345-357.

SKRAMLIK, E. voN (1924). Über die Lokalisation der Empfindungen bei den niederen Sinnen. Zeitschrift für Psychologie \& Physiologie Sinnesorgane, 56, 69-88.

STEVENS, J. C. (1991). Thermal sensibility. In M. A. Heller \& W. Schiff (Eds.), The psychology of touch (pp. 61-90). Hillsdale, NJ: Erlbaum.

Stevens, J. C. (1995). Detection of heteroquality taste mixtures. Perception \& Psychophysics, 57, 18-26.

Taus, R. H., Stevens, J. C.. \& Marks, L. E. (1975). Spatial localization of warmth. Perception \& Psychophysics, 17, 194-196.

Todrank, J., \& Bartoshuk, L. M. (1991). A taste illusion: Taste sensation localized by touch. Physiology \& Behavior, 50, 1027-1031.
Tomita, H., IKEDA, M., \& OKUdA, Y. (1986). Basis and practice of clinical taste examinations. Auris Nasus Larynx, 13, S1-15.

Travers, S. P., \& Norgren, R. (1995). Organization of orosensory responses in the nucleus of the solitary tract of the rat. Journal of Neurophysiology, 73, 2144-2162.

WEINSTEIN, S. (1968). Intensive and extensive aspects of tactile sensitivity as a function of body part, sex, and laterality. In D. R. Kenshalo (Ed.), The skin senses (pp. 195-222). Springfield, IL: Thomas.

Whitehead, M. C., \& KaChele, D. L. (1994). Development of the fungiform papillae, taste buds, and their innervation in the hamster. Journal of Comparative Neurology, 340, 515-530.

(Manuscript received June 25, 1998; revision accepted for publication May $3,1999$. 